\title{
Direct oral anticoagulants for the treatment of acute venous thromboembolism in patients with cancer: a meta-analysis of randomised controlled trials
}

\author{
To the Editor:
}

Venous thromboembolism (VTE) is a common complication in cancer patients $[1,2]$, associated with an $8-10 \%$ annual bleeding risk during anticoagulant therapy and a $10-20 \%$ annual risk of VTE recurrence $[1,3]$.

Although low-molecular-weight heparin (LMWH) is the recommended anticoagulant therapy for cancer-associated VTE [4, 5], vitamin-K antagonists (VKAs) are still frequently used [6, 7]. Previous meta-analyses assessed the safety and efficacy of direct oral anticoagulants (DOACs) in patients with cancer-associated VTE [8]. However, subgroup analyses provided additional evidence with separate data on patients with cancer history and on patients with active cancer, who were previously grouped together.

To compare the efficacy and safety of DOACs with VKAs for the treatment of VTE separately in these subgroups of patients, we extracted data from four post-hoc analyses of six randomised controlled trials (RE-COVER I, RE-COVER II, EINSTEIN-DVT, EINSTEIN-PE, AMPLIFY, Hokusai-VTE) [9-12]. Patients were classified as patients with active cancer, patients with a history of cancer, and patients without active or previous cancer. The primary efficacy outcome was recurrent VTE or VTE-related death. Safety outcome was major bleeding. Differences between the DOAC and VKA groups in the efficacy and safety outcomes were expressed as risk ratios with 95\% confidence intervals using a random-effects model. The significance level was set at $\mathrm{p}<0.05$. Heterogeneity among studies was assessed with Chi-squared Cochran's $\mathrm{Q}$ tests and with the $\mathrm{I}^{2}$ statistic.

Four studies [9-12] enrolled 13593 patients receiving DOACs and 13585 receiving VKAs. Overall, 1496 patients (5.5\%) had active cancer and 1605 (5.9\%) had cancer history. The proportion of patients with active cancer was similar in the DOAC and VKA groups ( $5.6 \%$ versus $5.2 \%, \mathrm{p}=0.11$ ) as well as the proportion of patients with cancer history $(7.2 \%$ versus $7.3 \%, \mathrm{p}=0.77)$. The risk of recurrent VTE was higher in patients with active cancer than in patients with cancer history (RR 1.78, 95\% CI 1.3-2.4) and in patients without cancer (RR 3.2, 95\% CI 2.6-3.9). Similarly, the risk of major bleeding was higher in patients with active cancer than in patients with cancer history (RR 1.79, 95\% CI 1.2-2.7) and in patients without cancer (RR 3.3, 95\% CI 2.5-4.4).

In patients with active cancer, DOACs were associated with a significantly lower risk of VTE recurrence (figure 1) and a nonsignificantly lower risk of major bleeding (figure 2) compared with VKAs.

In patients with cancer history, DOACs were associated with a nonsignificantly lower risk of VTE recurrence and major bleeding (figures 1 and 2) compared with VKAs.

In patients without cancer, the incidence of VTE recurrence was similar between DOACs and VKAs (figure 1), whereas DOACs were associated with a significantly lower risk of major bleeding (figure 2) than VKAs.

@ERSpublications

DOACs have a marginally higher antithrombotic efficacy and a comparable safety than VKA in cancer patients with VTE http://ow.ly/5lJN30dVVGF

Cite this article as: Di Minno MND, Ageno W, Lupoli R, et al. Direct oral anticoagulants for the treatment of acute venous thromboembolism in patients with cancer: a meta-analysis of randomised controlled trials. Eur Respir J 2017; 50: 1701097 [https://doi.org/10.1183/13993003.01097-2017]. 


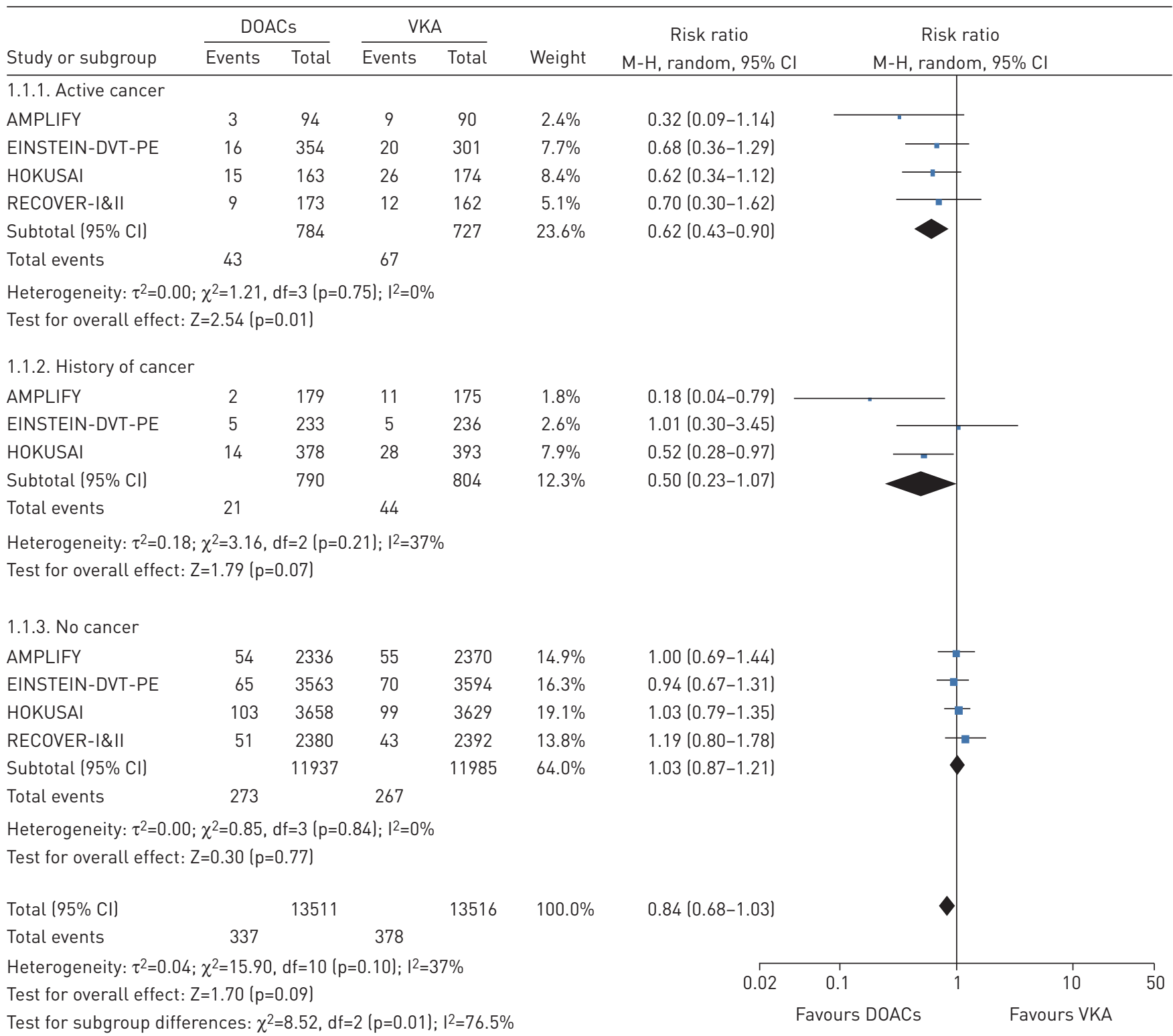

FIGURE 1 Risk of venous thromboembolism (VTE) recurrence and VTE-related mortality in patients receiving direct oral anticoagulants (DOACs) and vitamin $\mathrm{K}$ antagonists (VKAs) stratified according to the presence of cancer.

The risk of mortality was comparable in patients allocated to DOACs or VKAs: $18.0 \%$ versus $18.5 \%$ in patients with active cancer (RR 0.97 , 95\% CI 0.8-1.2), 7.4\% versus $7.0 \%$ in patients with cancer history (RR 1.06, 95\% CI 0.7-1.6), and $1.2 \%$ versus $1.2 \%$ in patients without cancer (RR 0.95, 95\% CI 0.7-1.2).

This is the first meta-analysis comparing separately the safety and efficacy of DOACs with VKAs for acute VTE in patients with active cancer, patients with a history of cancer, and patients without active or previous cancer. Our results indicate that DOACs are associated with a significantly lower risk of recurrent VTE than VKAs in patients with active cancer, while the risk of bleeding and mortality is similar between the treatment groups. These findings show striking similarities with the results of two Cochrane reviews [5, 13], reporting a significant reduction in the incidence of recurrent VTE in patients receiving LMWH compared with those receiving VKAs, with no differences in bleeding or survival. Accordingly, LMWH is the treatment of choice in cancer-associated VTE [4] because of fewer drug-drug interactions (especially with chemotherapeutic agents), a more predictable dose response, no need for laboratory monitoring, reliable delivery in patients with nausea/vomiting and a shorter half-life allowing a greater flexibility during invasive procedures and in cases where chemotherapy-induced thrombocytopenia develops. Many of these advantages are also offered by DOACs, with the addition of the oral route of administration, 


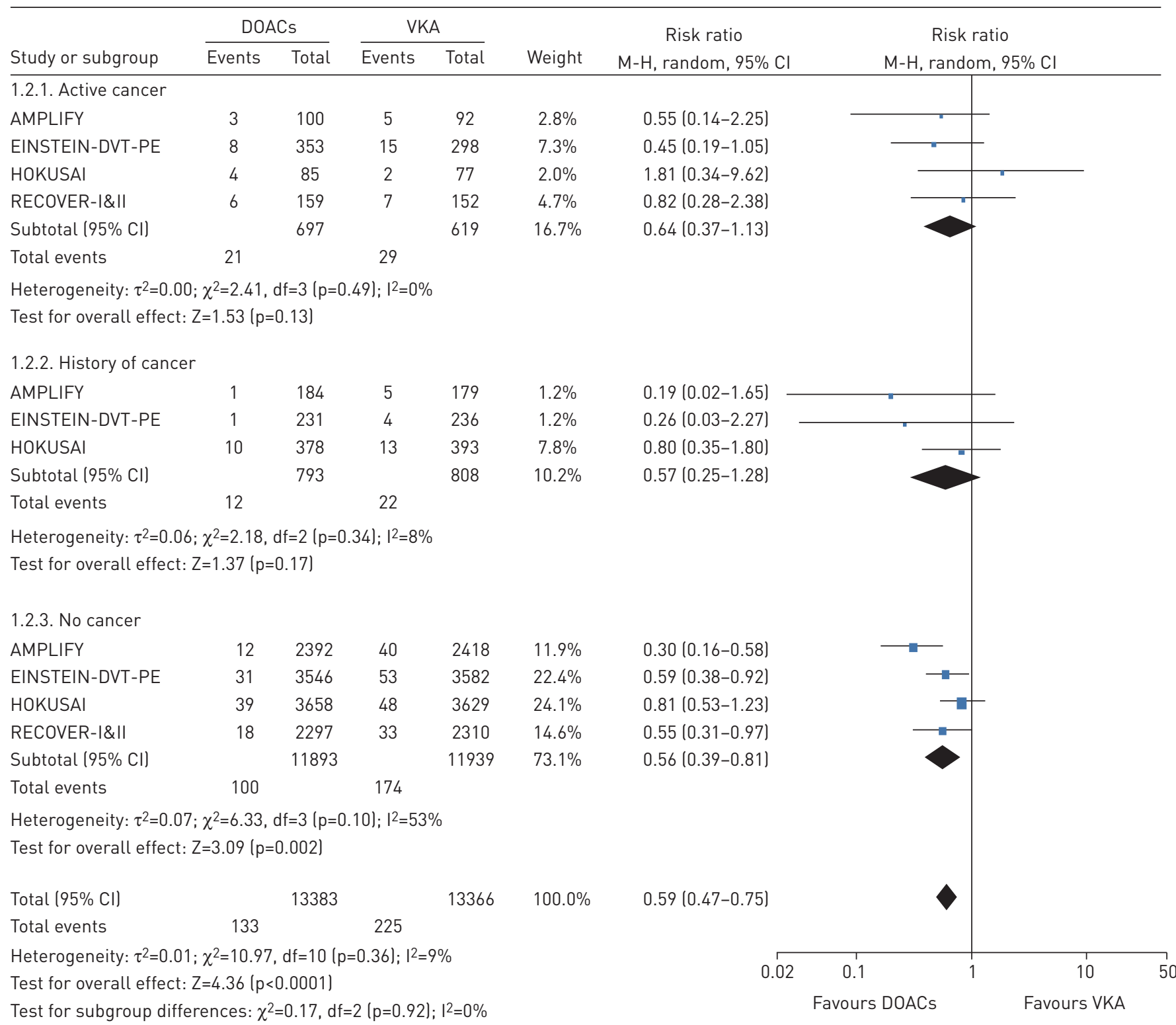

FIGURE 2 Risk of major bleeding in patients receiving direct oral anticoagulants (DOACs) and vitamin K antagonists (VKAs) stratified according to the presence of cancer.

which makes these agents more appealing for the long-term secondary prevention of VTE. However, before DOACs can be routinely proposed for patients with cancer-associated VTE, direct head-to-head comparison with LMWH is necessary. Indeed, in most of the available randomised controlled trials, the assessment of the safety and efficacy of DOACs in the subgroup of cancer patients was not pre-specified and patients with reduced life expectancy and end-organ dysfunction (e.g. renal and liver dysfunction) were excluded. Furthermore, anticipated treatment with LMWH was an explicit exclusion criterion in some studies. Consequently, patients with active cancer included in these trials had significantly lower rates of mortality, VTE recurrence, and bleeding than those reported in previous trials comparing LMWH versus VKAs [5]. Thus, the results of the present study may not apply, for instance, to cancer patients with advanced stage disease [1].

As an additional point, information from currently available trials is insufficient to fully appreciate potential concerns regarding the safety of DOACs in cancer patients receiving chemotherapy, due to potential interactions with antineoplastic agents [14]. Ongoing studies such as the Hokusai VTE-cancer study, in which enrolment has now been completed, and the Caravaggio study, for which enrolment is about to commence, will address this important knowledge gap [15]. 
In some previous studies, patients with active cancer and patients with history of cancer have been initially analysed together, under the assumption that the risk of adverse events could be similar between the two groups. The results of our study clearly show that both recurrence and bleeding rates are higher in the former group than in the latter, suggesting that these patients may require different management approaches.

In patients with a history of cancer, DOACs seem to have a favourable risk-benefit profile compared with VKAs, although the differences did not reach statistical significance in this study. In particular, the magnitude of the reduction in major bleeding rates was similar to that obtained in patients with active cancer and in patients without cancer; the lack of statistical significance may be driven by the lower rate of major bleeding complications.

The present study has potential limitations. First, we performed a meta-analysis of aggregate data, and difference in the baseline characteristics in patients treated with DOACs and VKAs could not be excluded since, in most studies, patients were not stratified based on the presence of cancer. Second, one could question the validity of pooling together the results of single studies, since the studies did not use the same definition of active cancer. However, as revealed by the $\mathrm{I}^{2}$ values (figures 1 and 2 ), heterogeneity among the studies in active cancer patients was low in our random-effects meta-analysis. Third, owing to the low number of included studies, we were unable to assess the presence of publication bias. However, it is unlikely that other trials outside the phase 3 programmes have been performed in which DOACs were compared with VKAs.

In conclusion, the results of the present meta-analysis suggest a favourable risk-benefit ratio for the use of DOACs in cancer patients with acute VTE when compared with the use of VKAs. Several safety aspects of the use of DOACs in cancer patients need additional evaluation. Ongoing studies that compare LMWH with DOACs for the treatment of VTE in cancer patients will address important knowledge gaps.

Matteo Nicola Dario Di Minno', Walter Ageno², Roberta Lupoli', Giulia Conte ${ }^{2}$, Nick van Es ${ }^{3}$, Harry R. Buller and Francesco Dentali $\oplus^{2}$

${ }^{1}$ Dept of Advanced Biomedical Sciences, Division of Cardiology, Federico II University, Naples, Italy. ${ }^{2}$ Dept of Clinical and Experimental Medicine, University of Insubria, Varese, Italy. ${ }^{3}$ Dept of Vascular Medicine, Academic Medical Center, Amsterdam, The Netherlands.

Correspondence: Matteo Nicola Dario Di Minno, Dept of Clinical Medicine and Surgery, Federico II University, Via S. Pansini 5, 80131 Napoli, Italy. E-mail: dario.diminno@hotmail.it

Received: April 052017 | Accepted after revision: June 212017

Acknowledgements: Author contributions: M.N.D. Di Minno conceived and designed the study, performed the analysis, interpreted results and drafted the manuscript; F. Dentali conceived and designed the study, and performed literature searches, data extraction and manuscript drafting; R. Lupoli and G. Conte performed literature searches, data extraction and manuscript drafting; N. van Es, H. Büller and W. Ageno interpreted results and performed critical revision of the manuscript. All authors read and approved the final version of the manuscript.

Conflict of interest: Disclosures can be found alongside this article at erj.ersjournals.com

\section{References}

1 Brose KMJ, Lee AYY. Cancer-associated thrombosis: prevention and treatment. Curr Oncol 2008; 15: Suppl. 1, 58-67.

2 Agnelli G, Verso M. Management of venous thromboembolism in patients with cancer. J Thromb Haemost 2011; 9: Suppl. 1, 316-324.

3 Francis CW, Kessler CM, Goldhaber SZ, et al. Treatment of venous thromboembolism in cancer patients with dalteparin for up to 12 months: the DALTECAN Study. J Thromb Haemost 2015; 13: 1028-1035.

4 Farge D, Bounameaux H, Brenner B, et al. International clinical practice guidelines including guidance for direct oral anticoagulants in the treatment and prophylaxis of venous thromboembolism in patients with cancer. Lancet Oncol 2016; 17: 452-466.

5 Akl EA, Kahale L, Barba M, et al. Anticoagulation for the long-term treatment of venous thromboembolism in patients with cancer. Cochrane Database Syst Rev 2014: CD006650.

6 Ageno W, Samperiz A, Caballero R, et al. Duration of anticoagulation after venous thromboembolism in real world clinical practice. Thromb Res 2015; 135: 666-672.

7 Lyman GH, Khorana AA, Kuderer NM, et al. Venous thromboembolism prophylaxis and treatment in patients with cancer: American Society of Clinical Oncology clinical practice guideline update. J Clin Oncol 2013; 31: 2189-2204.

8 van der Hulle T, den Exter PL, Kooiman J, et al. Meta-analysis of the efficacy and safety of new oral anticoagulants in patients with cancer-associated acute venous thromboembolism. J Thromb Haemost 2014; 12: $1116-1120$.

9 Schulman S, Goldhaber SZ, Kearon C, et al. Treatment with dabigatran or warfarin in patients with venous thromboembolism and cancer. Thromb Haemost 2015; 114: 150-157.

10 Prins $\mathrm{MH}$, Lensing AW, Brighton TA, et al. Oral rivaroxaban versus enoxaparin with vitamin $\mathrm{K}$ antagonist for the treatment of symptomatic venous thromboembolism in patients with cancer (EINSTEIN-DVT and 
EINSTEIN-PE): a pooled subgroup analysis of two randomised controlled trials. Lancet Haematol 2014; 1: e37-e46.

11 Raskob GE, van Es N, Segers A, et al. Edoxaban for venous thromboembolism in patients with cancer: results from a non-inferiority subgroup analysis of the Hokusai-VTE randomised, double-blind, double-dummy trial. Lancet Haematol 2016; 3: e379-e387.

12 Agnelli G, Buller HR, Cohen A, et al. Oral apixaban for the treatment of venous thromboembolism in cancer patients: results from the AMPLIFY trial. J Thromb Haemost 2015; 13: 2187-2191.

13 Akl EA, Vasireddi SR, Gunukula S, et al. Anticoagulation for the initial treatment of venous thromboembolism in patients with cancer. Cochrane Database Syst Rev 2014: CD006649.

14 Borges-Walmsley MI, McKeegan KS, Walmsley AR. Structure and function of efflux pumps that confer resistance to drugs. Biochem J 2003; 376: 313-338.

15 van Es N, Di Nisio M, Bleker SM, et al. Edoxaban for treatment of venous thromboembolism in patients with cancer. Rationale and design of the Hokusai VTE-cancer study. Thromb Haemost 2015; 114: 1268-1276. 